This is an electronic reprint of the original article. This reprint may differ from the original in pagination and typographic detail.

Author(s): Banichuk, Nikolay; Barsuk, Alexander; Ivanova, Svetlana; Makeev, Evgeni;

Neittaanmäki, Pekka; Tuovinen, Tero

Title: $\quad$ Analysis and optimization against buckling of beams interacting with elastic foundation

Year: $\quad 2018$

Version:

Please cite the original version:

Banichuk, N., Barsuk, A., Ivanova, S., Makeev, E., Neittaanmäki, P., \& Tuovinen, T. (2018). Analysis and optimization against buckling of beams interacting with elastic foundation. Mechanics Based Design of Structures and Machines, 46(5), 615-633. https://doi.org/10.1080/15397734.2017.1377619

All material supplied via JYX is protected by copyright and other intellectual property rights, and duplication or sale of all or part of any of the repository collections is not permitted, except that material may be duplicated by you for your research use or educational purposes in electronic or print form. You must obtain permission for any other use. Electronic or print copies may not be offered, whether for sale or otherwise to anyone who is not an authorised user. 


\section{Mechanics Based Design of Structures and Machines}

An International Journal

\section{Analysis and optimization against buckling of beams interacting with elastic foundation}

Nikolay Banichuk, Alexander Barsuk, Svetlana Ivanova, Evgeni Makeev, Pekka Neittaanmäki \& Tero Tuovinen

To cite this article: Nikolay Banichuk, Alexander Barsuk, Svetlana Ivanova, Evgeni Makeev, Pekka Neittaanmäki \& Tero Tuovinen (2017): Analysis and optimization against buckling of beams interacting with elastic foundation, Mechanics Based Design of Structures and Machines, DOI: 10.1080/15397734.2017.1377619

To link to this article: https://doi.org/10.1080/15397734.2017.1377619

Accepted author version posted online: 20 Oct 2017.

Submit your article to this journal

Џ Article views: 23

Q View related articles $₫$

View Crossmark data $\asymp$ 


\title{
Analysis and optimization against buckling of beams interacting with elastic foundation
}

\author{
Nikolay Banichuk, Alexander Barsuk, Svetlana Ivanova, Evgeni Makeev, Pekka Neittaanmäki,
} and Tero Tuovinen

\begin{abstract}
We consider an infinite continuous elastic beam that interacts with linearly elastic foundation and is under compression. The problem of the beam buckling is formulated and analyzed. Then the optimisation of beam against buckling is investigated. As a design variable (control function) we take the parameters of cross-section distribution of the beam from the set of periodic functions and transform the original problem of optimisation of infinite beam to the corresponding problem defined at the finite interval. All investigations are on the whole founded on the analytical variational approaches and the optimal solutions are studied as a function of problems parameters.
\end{abstract}

KEYWORDS: beams; buckling; elastic foundation; optimization; variational approach

Received 28 March 2017; Accepted 6 September 2017

Tero Tuovinen tero.tuovinen@jyu.fi 


\section{INTRODUCTION}

The model of beam resting on elastic foundation is very important as from theoretical as from engineering point of view for example in paper making processes. The value of this model is that it is the simplest one describing the interaction of elastic beam and external media and admits analytical investigations in many interesting practical cases. Reader can study a historical outline of the basic ideas in the theory of beams and plates resting on elastic foundation, described by Winkler law and contained in the books Timoshenko and Gere (1961); Timoshenko and Woinowsky-Krieger (1959).

The models of beams, interacting with elastic foundation, are often used for analysis of vibrations and stability. Static forms of elastic stability, developed by Euler (1766) for a differential equation describing the bending of a beam or column, were analyzed in Yayli (2017). The stability analysis of discrete models of compressed beams in elastic media is considered in De Angelis (2012). The influence of the elastic medium stiffness in the buckling behaviour of compressed beams on elastyic foundation is treated by De Angelis and Cancellara (2012). Analytical and numerical observations for buckling problems for beams on elastic foundation are presented by Griffiths and Bee (2014). Thermal bucling of beams on elastic foundation is considered in Vaz et al. (2014). Vibrations analysis of a continuous beam on elastic supports was presented in Banichuk et al. (2016). Special case of buckling and vibration is devoted infinite beams lying on elastic foundation. Corresponding methodology for the static analysis of infinite periodic structures under general loading was described in Moses et al. (2001). Stability of a beam with periodic supports was investigated in Avetisyan et al. (2015). Note also the paper by Soldatos and Selvadurai (1985) 
devoted the static problem analysis of the flexure of a beam resting on a nonlinear Winkler-type foundation.

In this paper we consider an infinite continuous elastic beam that interact with elastic foundation and formulate and solve the beam optimisation against buckling. As a result, we conclude that the optimal thickness distribution maximizing the critical buckling load of infinite beam resting on elastic foundation is a periodic structure with the period equal to the critical length of the finite compressed beam lying on elastic foundation with simply supported ends and optimal thickness distribution.

\section{ANALYSIS OF VARIATIONAL PROBLEMS DEPENDING ON SCALAR PARAMETERS}

Many variational problems, including buckling problem for a beam resting on a linearly elastic foundation, can be formulated as the following variational problem

$\psi(\gamma)=\min _{w} J(w ; \gamma)$

where $J(w, \gamma)$ is the problem functional depending on the system state function $w$ and a real scalar parameter $\gamma$. It is supposed that the state variable is differentiable function satisfying some boundary conditions. 
Let us obtain the expression for the first derivative $\mathrm{d} \psi(\gamma) / \mathrm{d} \gamma$. To this purpose we will use the formula

$\frac{\mathrm{d} \psi(\gamma)}{\mathrm{d} \gamma}=\lim _{\Delta \gamma \rightarrow 0} \frac{\psi(\gamma+\Delta \gamma)-\psi(\gamma)}{\Delta \gamma}$

Let solutions $w(\gamma)$ and $w(\gamma+\Delta \gamma)$ of variational problem in the equation 1 correspond to the values $\gamma$ and $\gamma+\Delta \gamma$ and thus we have

$\delta_{w} J(w(\gamma) ; \gamma)=0$

$\delta_{w} J(w(\gamma+\Delta \gamma) ; \gamma+\Delta \gamma)=0$

$\psi(\gamma)=J(w ; \gamma), \quad \psi(\gamma+\Delta \gamma)=J(w(\gamma+\Delta \gamma) ; \gamma+\Delta \gamma)$ 
Taking into account the equations (1)-(5) we transform the expression for the first derivate of $\psi(\gamma)$ as

$$
\begin{aligned}
\frac{\mathrm{d} \psi(\gamma)}{\mathrm{d} \gamma} & =\lim _{\Delta \gamma \rightarrow 0} \frac{J(w(\gamma+\Delta \gamma) ; \gamma+\Delta \gamma)-J(w(\gamma) ; \gamma)}{\Delta \gamma} \\
& =\lim _{\Delta \gamma \rightarrow 0} \frac{J(w(\gamma)+\Delta w(\gamma) ; \gamma+\Delta \gamma)-J(w(\gamma) ; \gamma)}{\Delta \gamma} \\
& =\lim _{\Delta \gamma \rightarrow 0} \frac{\delta_{w} J(w(\gamma) ; \gamma)+\frac{\partial J}{\partial \gamma} \Delta \gamma}{\Delta \gamma}=\frac{\partial J}{\partial \gamma} .
\end{aligned}
$$

Thus we arrive the following representation for $\mathrm{d} \psi(\gamma) / \mathrm{d} \gamma$ in the form

$$
\frac{\mathrm{d} \psi(\gamma)}{\mathrm{d} \gamma}=\frac{\partial J(w ; \gamma)}{\partial \gamma}
$$

In the case, when $\gamma_{1} \leq \gamma \leq \gamma_{2}$ and $\mathrm{d} \psi(\gamma) / \mathrm{d} \gamma \neq 0$, where $\gamma_{1}$ and $\gamma_{2}$ are given values, the dependence $\psi(\gamma)$ is monotonic and achieves its extreme values at $\gamma=\gamma_{1}$ and $\gamma=\gamma_{2}$.

Let $\gamma=\gamma_{*}$ is the value of the problem parameter for which $\mathrm{d} \psi(\gamma) / \mathrm{d} \gamma=0$. According to the equation (7) we have the following condition

$\frac{\partial J(w ; \gamma)}{\partial \gamma}=0$

for determination of $\gamma_{*}$. Note that the equation (8) is a nonlinear with respect to $\gamma$ and its solution can be considered as some functional defined on the solution $w$. Let us denote the solution of equation (8) (one from solutions if there exist several solutions) by $\gamma_{*}=\gamma(w)$ and the dependence $\psi(\gamma)$ corresponding $\gamma=\gamma_{*}$, by $\psi_{*}=\psi\left(\gamma_{*}\right)$. 
It is possible to show that the following variational representation

$\psi_{*}=\min _{w} J(w ; \gamma(w))=\min _{w} \Phi(w)$,

$\Phi(w) \equiv J(w ; \gamma(w))$

is valid. In fact, taking into account equations (3)-(5) and (8) we obtain

$\delta \Phi(w)=\delta_{w} J(w ; \gamma(w))+\frac{\partial J}{\partial \gamma} \delta \gamma(w)=0$

and consequently the first variation of functional $\Phi(w)$ vanish (reduces zero) at $\gamma=\gamma_{*}$.

Variational formulation (9) and (10) will be essentially used for analysis of considered problems and can be taken as a basis for obtaining variational estimates of $\psi_{*}$.

\section{STABILITY OF BEAMS WITH CONSTANT STIFFNESS PARAMETERS}

To analyze the stability problem of continuous beam of periodic mechanical and geometrical characteristics distribution and periodic boundary conditions let us consider an elastic beam of finite length $\ell$ and volume $V$ resting on a linearly elastic foundation, characterizing by rigidity coefficient $c$. The beam has constant (along the beam) bending rigidity $E I$ and is subjected to compression by axial force $P$. The beam endpoints are simply supported at $x=0$ and $x=\ell$ (see schematic Figure 1). The governing equation of buckling (loss of the stability) and boundary conditions for deflection function $w(x)$, describing the transverse beam displacements in the interval $0<x<\ell$, 
are

$E I \frac{\mathrm{d}^{4} w}{\mathrm{~d} x^{4}}+P \frac{\mathrm{d}^{2} w}{\mathrm{~d} x^{2}}+c w=0$

$w(0)=E I\left(\frac{\mathrm{d}^{2} w}{\mathrm{~d} x^{2}}\right)_{x=0}=w(\ell)=E I\left(\frac{\mathrm{d}^{2} w}{\mathrm{~d} x^{2}}\right)_{x=\ell}=0$

where $E$ is Young modulus, $I$ is moment of inertia of the beam's cross-section. The reaction of the considered elastic foundation is proportional to the deflection of the beam at that point, that is, the beam reaction can be represented by Hooke's law, $c w$. Such theoretical model for beams resting on elastic foundation are adequate to a wide class of engineering problems stipulated by buckling phenomena and have been discussed in the classical literature (see e.g. the books by Timoshenko and Woinowsky-Krieger (1959) and von Kármán and Biot (1940)).

For convenience of comparative analysis of solutions of spectral problem (12) and (13) with constant bending rigidities $E I$ and analogous problem with varying bending rigidities $E I(x)$ we introduce the bending rigidity distribution of the form

$E I=A_{j} S^{j}, \quad j=1,2,3$

where $S$ is cross-sectional area, and the coefficients $A_{1}, A_{2}$ and $A_{3}$ characterize the type of crosssection. In this connection $j=0$, corresponding to the bending rigidity $E I=A_{0}$, is not related with the cross-section shape characteristics. Thus, all obtained relations for $j=0$ correspond to generally accepted relations for beams with constant bending rigidity distribution. 
Let us introduce dimensionless independent variable $\tilde{x}$, normalized cross-section area $\tilde{S}$, parameter $\lambda$ and $\tilde{c}$ (in what follows tilde is omitted)

$x=\ell \tilde{x}, \quad S=\frac{V \tilde{S}}{\ell}, \quad P=A_{j} V^{j} \lambda$,

$c=A_{j} V^{j} \tilde{c}, \quad j=0,1,2,3$.

and rewrite spectral boundary-value problem as

$\frac{\mathrm{d}^{4} w}{\mathrm{~d} x^{4}}+\lambda \ell^{j+2} \frac{\mathrm{d}^{2} w}{\mathrm{~d} x^{2}}+c \ell^{j+4} w=0$

$w(0)=\left(\frac{\mathrm{d}^{2} w}{\mathrm{~d} x^{2}}\right)_{x=0}=w(1)=\left(\frac{\mathrm{d}^{2} w}{\mathrm{~d} x^{2}}\right)_{x=1}=0$.

Note that the stability equation is formulated in the form (16) to represent the dependence on the beam length in the explicit manner. Solutions of the boundary-value problem (16) and (17) are found with the help of Fourier series

$w(x)=\sum_{n=1}^{\infty} c_{n} \sin n \pi x$.

Using equation (18) we transform equations (16) and (17) into the following equation

$\sum_{n=1}^{\infty} c_{n}\left((n \pi)^{4}-(n \pi)^{2} \lambda \ell^{j+2}+c \ell^{j+4}\right) \sin n \pi x=0$. 
Taking into account the properties of completeness and orthogonality of the system $\sin n \pi x$, where $n=1,2,3, \ldots$, we conclude that the equation (19) is fulfilled when all coefficients in the equation (19) vanish, i.e.

$c_{n}\left((n \pi)^{4}-(n \pi)^{2} \lambda \ell^{j+2}+c \ell^{j+4}\right)=0, \quad n=1,2,3, \ldots$

Vanishing of the first multiplier $c_{n}$ in the equation (20) means the exclusion of the corresponding Fourier term from the equation (18), but the vanishing of the second multiplier in the equation (20) gives us the relation

$(n \pi)^{4}-(n \pi)^{2} \lambda \ell^{j+2}+c \ell^{j+4}=0$

between the parameters $\lambda, c$ and $\ell$. We can also write this relation as

$\lambda_{n}(\ell)=\frac{n^{2} \pi^{2}}{\ell^{j+2}}+c \frac{\ell^{2}}{n^{2} \pi^{2}}, \quad n=1,2,3, \ldots$

Critical force of buckling corresponds to minimal value of $\lambda_{n}(\ell)$, which is found for appropriate meaning of $n$.

If we fix some number $n$ and consider the buckling phenomenon with formation of $n$ half-waves than we can determine the critical value of beam length $\ell$ minimizing $\lambda_{n}(\ell)$ and satisfying the conditions

$\frac{\mathrm{d} \lambda_{n}(\ell)}{\mathrm{d} \ell}=0$ 
Using equation (23) we obtain the expression for critical lengths $\ell_{n}^{*}$ and corresponding critical buckling forces $\lambda_{n}^{*}=\lambda_{n}\left(\ell_{n}^{*}\right)$ :

$\ell_{n}^{*}=\left(\frac{j+2}{2 c}\right)^{\frac{1}{j+4}}(n \pi)^{\frac{4}{j+4}}, \quad n=1,2,3, \ldots$

$\lambda_{n}^{*}=\frac{j+4}{2}\left(\frac{2 c}{j+2}\right)^{\frac{j+2}{j+4}}(n \pi)^{\frac{-2 j}{j+4}}$

In particular case, when $j=0$, from the equation (24) it follows that

$\ell_{n}^{*}=\frac{n \pi}{c^{1 / 4}}, \quad \lambda_{n}^{*}=2 \sqrt{c}, \quad n=1,2,3, \ldots$

As it is seen from the equation (25), the decreasing of the foundation stiffness corresponds to increase of critical beam length and the critical force $\lambda_{n}^{*}$ (for this length) does not depend on the number of half-waves.

\section{VARIATIONAL PROBLEM FOR THE BEAMS OF CRITICAL LENGTH}

Differential equation (12) is a necessary extremum condition, i.e. Euler differential equation, for nonadditive functional

$$
P=\min _{w} \frac{\int_{0}^{\ell} E I\left(\frac{\mathrm{d}^{2} w}{\mathrm{~d} x^{2}}\right)^{2} \mathrm{~d} x+c \int_{0}^{\ell} w^{2} \mathrm{~d} x}{\int_{0}^{\ell}\left(\frac{\mathrm{d}^{2} w}{\mathrm{~d} x^{2}}\right)^{2} \mathrm{~d} x} .
$$


Using notations from equation (15) the variational problem of elastic stability (12), (13) and (26) is written as

$\lambda(\ell)=\min _{w}\left\{\frac{1}{\ell^{j+2}} \frac{J_{1}(w)}{J_{3}(w)}+c \ell^{2} \frac{J_{2}(w)}{J_{3}(w)}\right\}$

$w(0)=\left(\frac{\mathrm{d}^{2} w}{\mathrm{~d} x^{2}}\right)_{x=0}=0, \quad w(1)=\left(\frac{\mathrm{d}^{2} w}{\mathrm{~d} x^{2}}\right)_{x=1}=0$

Here we use the following notations for introduced functional

$J_{1}(w)=\int_{0}^{1}\left(\frac{\mathrm{d}^{2} w}{\mathrm{~d} x^{2}}\right)^{2} \mathrm{~d} x$

$J_{2}(w)=\int_{0}^{1} w^{2} \mathrm{~d} x$

$J_{3}(w)=\int_{0}^{1}\left(\frac{\mathrm{d} w}{\mathrm{~d} x}\right)^{2} \mathrm{~d} x$

Note that for rigorous solutions

$w_{n}(x)=c_{n} \sin n \pi x, \quad n=1,2,3, \ldots$ 
of the variational problem (27)-(28) we have

$J_{1}\left(w_{n}\right)=\frac{n^{4} \pi^{4}}{2} c_{n}^{2}, \quad J_{2}\left(w_{n}\right)=\frac{1}{2} c_{n}^{2}, \quad J_{3}\left(w_{n}\right)=\frac{n^{2} \pi^{2}}{2} c_{n}^{2}$

After substitution of expressions (33) into equation (27), we obtain the expression (22) for $\lambda_{n}(\ell)$.

Note that the variational representation in the equation (27) is valid for the beams of arbitrary length and gives us the possibility to find corresponding critical buckling forces.

Let us transform the variational representation (27) to the form for the beams of critical length defined by the condition $\mathrm{d} \lambda(\ell) / \mathrm{d} \ell=0$. Using the rule of differentiation with respect to parameter (7) and formulas (9) and (10) we obtain the following representation for the beam of critical lengths $\ell^{*}=\left(\frac{j+2}{2 c}\right)^{\frac{1}{j+4}}\left(\frac{J_{1}(w)}{J_{2}(w)}\right)^{\frac{1}{j+4}}$

$\lambda^{*}=\frac{j+4}{2}\left(\frac{2 c}{j+2}\right)^{\frac{j+2}{j+4}} \min _{w} \Phi_{j}(w)$

$\Phi_{j}(w)=\frac{1}{J_{3}(w)}\left(J_{1}^{2}(w) J_{2}^{j+2}(w)\right)^{\frac{1}{j+4}}, \quad j=0,1,2,3$.

Here the deflection functions $w$ satisfies the boundary conditions (27). Necessary extremum condition $\delta \Phi_{j}(w)=0$ for the functional $\Phi_{j}(w)$ and the corresponding boundary conditions 
constitute the following spectral boundary value problem

$$
\begin{aligned}
& \frac{\mathrm{d}^{4} w}{\mathrm{~d} x^{4}}+a(w) \frac{\mathrm{d}^{2} w}{\mathrm{~d} x^{2}}+b(w) w=0 \\
& w(0)=\left(\frac{\mathrm{d}^{2} w}{\mathrm{~d} x^{2}}\right)_{x=0}=w(1)=\left(\frac{\mathrm{d}^{2} w}{\mathrm{~d} x^{2}}\right)_{x=1}=0
\end{aligned}
$$

where the functionals $a(w)$ and $b(w)$ are determined by the following expressions

$$
a(w)=\frac{j+4}{2} \frac{J_{1}(w)}{J_{3}(w)}, \quad b(w)=\frac{j+2}{2} \frac{J_{1}(w)}{J_{2}(w)} .
$$

We will find the solution of spectral boundary-value problem $(37)-(38)$ in the form of Fourier series (18). Substitution of Fourier series (18) into (37) and some elementary transformations give us the equation

$$
\sum_{n=1}^{\infty} c_{n}\left((n \pi)^{4}-a(w)(n \pi)^{2}+b(w)\right) \sin n \pi x=0
$$

Performing corresponding analysis we reduce the equation (40) to the relations between the functionals $a(w)$ and $b(w)$ :

$$
(n \pi)^{4}-a(w)(n \pi)^{2}+b(w)=0, \quad n=1,2,3, \ldots
$$

Note that the coefficients $c_{n} \neq 0$. It is seen that the functionals $J_{1}(w), J_{2}(w)$ and $J_{3}(w)$ have the following representation

$$
J_{1}(w)=\frac{1}{2} \sum_{n=1}^{\infty}(n \pi)^{4} c_{n}^{2}, \quad J_{2}(w)=\frac{1}{2} \sum_{n=1}^{\infty} c_{n}^{2}, \quad J_{3}(w)=\frac{1}{2} \sum_{n=1}^{\infty}(n \pi)^{2} c_{n}^{2} .
$$


In particular, when the deflection function $w$ is expressed by one component representation (32) with one harmonic function the considered functionals $J_{1}\left(w_{n}\right), J_{2}\left(w_{n}\right)$ and $J_{3}\left(w_{n}\right)$ take the values (33) and the functionals $a(w)$ and $b(w)$ are represented as

$a\left(w_{n}\right)=\frac{j+4}{2} n^{2} \pi^{2}, \quad b\left(w_{n}\right)=\frac{j+2}{2} n^{4} \pi^{4}, \quad n=1,2,3, \ldots$

The equations (41) are satisfied for $a(w)$ and $b(w)$ from (43) and $\forall n=1,2,3, \ldots$ and thus the functions $w_{n}(x)$, given by the equation (32), are the rigorous solution of the spectral problem (37)(38). For indicated rigorous solutions we will have the following values for the functional $\Phi_{j}(w)$, critical beam lengths and critical buckling forces when $n=1,2,3, \ldots$ and $j=0,1,2,3$ (see, Table 1).

\section{ANALYSIS AND STABILITY OPTIMIZATION FOR BEAMS WITH VARIABLE RIGIDITY CHARACTERISTICS}

\subsection{Variational problem}

Consider variational formulation of elastic stability problem for beam with variable rigidity characteristics resting on elastic foundation. For the beam of given length the problem of determination of critical buckling load is formulated as

$P=\min _{w} \frac{A_{j} \int_{0}^{\ell} S^{j}\left(\frac{\mathrm{d}^{2} w}{\mathrm{~d} x^{2}}\right)^{2} \mathrm{~d} x+c \int_{0}^{\ell} w^{2} \mathrm{~d} x}{\int_{0}^{\ell}\left(\frac{\mathrm{d} w}{\mathrm{~d} x}\right)^{2} \mathrm{~d} x}$

with boundary conditions

$w(0)=0, \quad\left(S^{j} \frac{\mathrm{d}^{2} w}{\mathrm{~d} x^{2}}\right)_{x=0}=0, \quad w(\ell)=0, \quad\left(S^{j} \frac{\mathrm{d}^{2} w}{\mathrm{~d} x^{2}}\right)_{x=\ell}=0$. 
Using dimensionless variables from (15) and notation

$m(x)=S^{j}(x)\left(\frac{\mathrm{d}^{2} w}{\mathrm{~d} x^{2}}\right), \quad j=1,2,3$

we rewrite the problem (44) as

$\lambda(S, \ell)=\min _{w}\left\{\frac{1}{\ell^{j+2}} \frac{J_{1}(S, w)}{J_{3}(S, w)}+c \ell^{2} \frac{J_{2}(w)}{J_{3}(w)}\right\}$

with conditions

$w(0)=m(0)=0, \quad w(1)=m(1)=0$

where $m=m(x)$ is the bending moment and the functionals $J_{1}, J_{2}$ and $J_{3}$ are given by the following expressions

$J_{1}(S, w)=\int_{0}^{1} S^{j}(x)\left(\frac{\mathrm{d}^{2} w}{\mathrm{~d} x^{2}}\right)^{2} \mathrm{~d} x=\int_{0}^{1} \frac{m^{2}(x)}{S^{j}(x)} \mathrm{d} x$

$J_{2}(w)=\int_{0}^{1} w^{2} \mathrm{~d} x$

$J_{3}(w)=\int_{0}^{1}\left(\frac{\mathrm{d} w}{\mathrm{~d} x}\right)^{2} \mathrm{~d} x$ 
Let us transform (47)-(48) for beams of critical lengths using condition $\mathrm{d} \lambda(\ell) / \mathrm{d} \ell=0$, or more detailed writing

$-\frac{j+2}{\ell^{j+3}} \frac{J_{1}}{J_{3}}+2 c \ell \frac{J_{2}}{J_{3}}=0$

As result we obtain the expressions for critical beam lengths and corresponding buckling loads

$\ell^{*}=\left(\frac{j+2}{2 c}\right)^{\frac{1}{j+4}}\left(\frac{J_{1}(S, w)}{J_{2}(w)}\right)^{\frac{1}{j+4}}, \quad j=0,1,2,3$,

$\lambda^{*}=\frac{j+4}{2}\left(\frac{2 c}{j+2}\right)^{\frac{j+2}{j+4}} \min _{w} \Phi_{j}(S, w)$

$\Phi_{j}(S, w)=\frac{1}{J_{3}(w)}\left(J_{1}^{2}(S, w) J_{2}^{j+2}(w)\right)^{\frac{1}{j+4}}$

The last expression for the functional $\Phi_{j}$ was derived with the help of (9). It is assumed here that the deflection functions satisfy the boundary conditions in (48). Necessary extremum condition for the functional $\Phi_{j}$ makes possible to formulate the boundary-value problem

$\frac{\mathrm{d}^{2}}{\mathrm{~d} x^{2}}\left(S^{j} \frac{\mathrm{d}^{2} w}{\mathrm{~d} x^{2}}\right)+a(S, w) \frac{\mathrm{d}^{2} w}{\mathrm{~d} x^{2}}+b(S, w) w=0$

$w(0)=\left(S^{j} \frac{\mathrm{d}^{2} w}{\mathrm{~d} x^{2}}\right)_{x=0}=w(1)=\left(S^{j} \frac{\mathrm{d}^{2} w}{\mathrm{~d} x^{2}}\right)_{x=1}=0$ 
which can be also written in the following form

$$
\frac{\mathrm{d}^{2} m}{\mathrm{~d} x^{2}}+a(S, w) \frac{\mathrm{d}^{2} w}{\mathrm{~d} x^{2}}+b(S, w) w=0
$$

$$
w(0)=m(0)=0, \quad w(1)=m(1)=0
$$

using the expressions for the moment (46) and the following expressions for the functionals $a$ and $b$ :

$$
a(S, w)=\frac{j+4}{2} \frac{J_{1}(S, w)}{J_{3}(w)}
$$

$$
b(S, w)=\frac{j+2}{2} \frac{J_{1}(S, w)}{J_{2}(w)} .
$$

\subsection{Optimization problem}

Consider the problem of cross-section area distribution maximizes the critical buckling load under beam volume constraints

$S^{*}(x): \quad \lambda_{*}(\ell)=\max _{S(x)} \lambda(S, \ell): \quad \int_{0}^{1} S(x) \mathrm{d} x=1$ 
where the functional $\lambda(S, \ell)$ is determined by the formulas (46), (47)and conditions (48). The requirement $\delta \lambda(S, \ell)=0$ gives us the necessary optimality condition

$m^{2}(x)=S^{\alpha+1}(x), \quad \alpha=1,2,3$

which is valid for arbitrary beam, including the case with $\ell=\ell^{*}$.

For critical lengths of beam the functional $\lambda^{*}(S)=\lambda\left(S, \ell^{*}\right)$ is determined by the equations (53)(55), (60), (61) and $\{(56),(57)\}$, or $\{(58),(59)\}$.

Necessary optimality conditions, the equation (63) with the equations (60), (61) and $\{(56),(57)\}$, or $\{(58),(59)\}$ constitutes the system for finding the optimal cross-sectional beam distribution.

\subsection{Some asymptotics}

In correspondence with the equation (63) and the boundary conditions $m(0)=m(1)=0$ (see, (59)) the optimal cross-section area is equal to zero at the beam ends, that vanish the coefficients for the highest derivative in the differential equation (56) at $x=0$ and $x=1$. In this context the spectral problem (56)-(57) with optimal solution $S^{*}(x)$ belongs to the singular perturbated spectral problems, which are required application of special analysis. To this purpose we investigate the asymptotic behaviour of optimal solutions in the vicinity of the beam-ends. Now we will consider the asymptotic behaviour at $x=0(x \ll 1)$. 
With application of equation (58)-(59) and necessary optimality condition (63) and also double integration of (58) with corresponding boundary conditions (59) we obtain the following integral equation

$m+a w+b \int_{0}^{x}(x-s) w(s)=C x, \quad C=b \int_{0}^{1}(1-s) w(s) \mathrm{d} s$.

Asymptotic behaviour of deflections and moments is found for $x \ll 1$ taking into account boundary conditions $w(0)=0$ and $m(0)=0$ as

$m(x) \approx C_{1} x^{\beta}, \quad w(x) \approx C_{2} x^{\gamma}, \quad \beta, \gamma>0$.

Using necessary optimality condition (63) and relations (65) we obtain the required asymptotic formulas

$S(x)=m^{2 / \alpha+1} \approx C_{1}^{\frac{2}{\alpha+1}} x^{\frac{2 \beta}{\alpha+1}}$

$w_{x x}(x)=\frac{m(x)}{S^{\alpha}(x)} \approx C_{1}^{-\frac{\alpha-1}{\alpha+1}} x^{-\frac{\alpha-1}{\alpha+1} \beta}$

\subsection{Analytical optimal solution for $\alpha=1$}

If we take into account necessary optimality condition (63) and the case $\alpha=1$ we present

$$
m^{2}(x)=S^{2}(x)\left(\frac{\mathrm{d}^{2} w(x)}{\mathrm{d} x^{2}}\right)^{2}=S^{2}(x)
$$


and nonlinear differential equation

$$
\left(\frac{\mathrm{d}^{2} w}{\mathrm{~d} x^{2}}\right)^{2}=1 \quad \text { or } \quad\left(\frac{\mathrm{d}^{2} w}{\mathrm{~d} x^{2}}\right)= \pm 1 .
$$

Using the boundary conditions we have

$$
w(x)=\frac{1}{2} x(1-x)
$$

and corresponding representation for the bending moment

$$
m(x)=S(x) \frac{\mathrm{d}^{2} w(x)}{\mathrm{d} x^{2}}=-S(x)
$$

Taking into account the previous relations we can write the differential equation and boundary conditions for $S(x)$ as

$-\frac{\mathrm{d}^{2} S}{\mathrm{~d} x^{2}}-a+\frac{1}{2} b x(1-x)=0, \quad S(0)=S(1)=0$

where $a$ and $b$ for $\alpha=1$ are determined by the expressions (see equations (60) and (61))

$$
a=\frac{5}{2} \frac{J_{1}}{J_{3}}, \quad b=\frac{3}{2} \frac{J_{1}}{J_{2}}
$$

In correspondence with the equation (70) the functional $J_{1}, J_{2}$ and $J_{3}$ (see equations (49)-(51)) are equal to

$$
J_{1}=1, \quad J_{2}=\frac{1}{120}, \quad J_{3}=\frac{1}{12}
$$


and consequently the functionals $a$, and $b$ and the solution of the optimization problem (72) are

$a=30, \quad b=180, \quad S(x)=\frac{15}{2} x(1-x)[1-x(1-x)]$.

Obtained optimal solution of cross-sectional areas is symmetric with respect to the middle point and archives the maximum value at this point:S(1/2) $=45 / 32$. Note that this optimal solution (75) does not depend on the basement rigidity. Optimal solution $S(x)$ and critical buckling force, corresponding to (53)-(55), are given by

$\ell^{*}=\left(\frac{3}{2 c} \frac{J_{1}}{J_{3}}\right)^{1 / 5}=\left(\frac{3}{2 c}\right)^{1 / 5} \cdot 2.60517$

$\lambda^{*}=\frac{5}{2 J_{3}}\left(\left(\frac{2 c}{3}\right)^{3} J_{1}^{2} J_{2}^{3}\right)^{1 / 5}=\frac{5}{2}\left(\frac{2 c}{3}\right)^{3 / 5} \cdot 0.678692$

For comparison we present also the values $\ell^{*}$ and $\lambda^{*}$ for the beam of constant cross-section $S(x)=1$ using the expressions (24) with $\alpha=1$ and $n=1$.

We have

$\ell^{*}=\left(\frac{3}{2 c}\right)^{1 / 5} \cdot 2.49873, \quad \lambda^{*}=\frac{5}{2}\left(\frac{2 c}{3}\right)^{3 / 5} \cdot 0.63262$

The shape of the optimal solution is presented in the Figure 2. 


\subsection{Numerical optimization solutions for $\alpha=2,3$}

Consider the relations (49)-(55) and the formulation of optimization problem (62) where the functional $\lambda(S, \ell)$ and the variational problem are determined as

$\lambda^{*}=\left(\frac{\alpha+4}{2}\right)\left(\frac{2 c}{\alpha+2}\right)^{\frac{\alpha+2}{\alpha+4}} \min _{w} \Phi_{\alpha}(S, w)$,

$\Phi_{\alpha}(S, w)=\frac{1}{J_{3}(w)}\left(J_{1}^{2}(S, w) J_{2}^{\alpha+2}(w)\right)^{\frac{1}{\alpha+4}}, \quad \alpha=1,2,3$

where the functionals $J_{1}, J_{2}$ and $J_{3}$ are determined by the equations (49)-(51).

The optimization problem as formulated in (62) with optimality conditions (63) give us the following relations

$m(x)=-a w(x)+b\left(x \int_{0}^{1}(1-\tau) w(\tau) \mathrm{d} \tau-\int_{0}^{x}(x-\tau) w(\tau) \mathrm{d} \tau\right)$

$w(x)=\int_{0}^{x} \frac{(x-\tau)}{S^{\alpha}(\tau)} \mathrm{d} \tau-x \int_{0}^{1} \frac{(1-\tau) m(\tau)}{S^{\alpha}(\tau)} \mathrm{d} \tau$

as a basis for the iteration procedure of finding the optimal solutions. Realization of iteration algorithm (which we omit) and performed the corresponding computations give us the following optimal solutions (see Figures 3 and 4). 


\section{OPTIMIZATION OF CONTINUOUS (INFINITE) BEAM WITH PERIODIC RIGIDITY CHARACTERISTIC}

\subsection{Stability of beam}

Consider the buckling problem for infinite elastic beam satisfied the following relation

$$
\begin{aligned}
& \frac{\mathrm{d}^{2}}{\mathrm{~d} x^{2}}\left(E I(x) \frac{\mathrm{d}^{2} w(x)}{\mathrm{d} x^{2}}\right)+P \frac{\mathrm{d}^{2} w(x)}{\mathrm{d} x^{2}}+c w(x)=0 \\
& |w(x)| \leq C \neq \infty, \quad-\infty<x<\infty \\
& E I(x) \equiv E I(x+n \ell), \quad n=0, \pm 1, \pm 2, \ldots
\end{aligned}
$$

where $\ell$ is the period.

The fundamental property of periodic spectral problem consists in

$$
\begin{aligned}
& m^{k+1}\left(x_{k}+x\right)=e^{i \alpha \ell} m^{k}\left(x_{k-1}+x\right), \quad 0 \leq x \leq \ell \\
& k=0, \pm 1, \pm 2, \ldots \quad 0 \leq \alpha \ell<2 \pi .
\end{aligned}
$$

The $x$-axis is divided in the equal intervals by points $x_{k-1}=(k-1) \ell$ (left end) and $x_{k}=k \ell$ (right end) $k=0, \pm 1, \pm 2, \ldots$, while

$$
E I\left(x_{k}+x\right)=E I\left(x_{k-1}+x\right)
$$


and

$m^{k+1}\left(x_{k}+x\right)=e^{i \alpha \ell} m^{k}\left(x_{k-1}+x\right)$.

The upper index in the equation (86) corresponds to the interval number.

Taking into account the continuity of variables

$w(x), \quad \frac{\mathrm{d} w(x)}{\mathrm{d} x}, \quad m(x), \quad \frac{\mathrm{d} m(x)}{\mathrm{d} x}$

at the nodes $x_{k}$, we can write the considered spectral problem for periodic intervals as

$\frac{\mathrm{d}^{2}}{\mathrm{~d} x^{2}}\left(E I(x) \frac{\mathrm{d}^{2} w(x)}{\mathrm{d} x^{2}}\right)+P \frac{\mathrm{d}^{2} w(x)}{\mathrm{d} x^{2}}+c w(x)=0, \quad 0<x<\ell$

$w(\ell)=e^{i \alpha \ell_{w}(0),} \quad\left(\frac{\mathrm{d} w}{\mathrm{~d} x}\right)_{x=\ell}=e^{i \alpha \ell}\left(\frac{\mathrm{d} w}{\mathrm{~d} x}\right)_{x=0}$,
$m(\ell)=e^{i \alpha \ell} m(0), \quad\left(\frac{\mathrm{d} m}{\mathrm{~d} x}\right)_{x=\ell}=e^{i \alpha \ell}\left(\frac{\mathrm{d} m}{\mathrm{~d} x}\right)_{x=0} \quad 0 \leq \alpha \leq 2 \pi$

or in dimensionless variables

$x=\ell \tilde{x}, \quad S=\frac{V \tilde{S}}{\ell}, \quad P=A_{j} V^{j} \lambda$

$C=A_{j} V^{j} \tilde{C}, \quad \alpha \ell=\tilde{\alpha}, \quad j=1,2,3$. 
(Tilde is omitted) as

$$
\begin{aligned}
& \frac{\mathrm{d}^{2}}{\mathrm{~d} x^{2}}\left(S^{j}(x) \frac{\mathrm{d}^{2} w}{\mathrm{~d} x^{2}}\right)+\lambda \ell^{j+2} \frac{\mathrm{d}^{2} w}{\mathrm{~d} x^{2}}+c \ell^{j+4} w=0, \quad 0<x<1 \\
& w(1)=e^{i \alpha} w(0), \quad\left(\frac{\mathrm{d} w}{\mathrm{~d} x}\right)_{x=1}=e^{i \alpha}\left(\frac{\mathrm{d} w}{\mathrm{~d} x}\right)_{x=0} \\
& m(1)=e^{i \alpha} m(0), \quad\left(\frac{\mathrm{d} m}{\mathrm{~d} x}\right)_{x=1}=e^{i \alpha}\left(\frac{\mathrm{d} m}{\mathrm{~d} x}\right)_{x=0} \quad 0 \leq \alpha \leq 2 \pi
\end{aligned}
$$

where $E I(x)=A_{j} S^{j}(x), j=1,2,3$.

In what follows we will also use the complex conjugate system

$$
\begin{aligned}
& \frac{\mathrm{d}^{2}}{\mathrm{~d} x^{2}}\left(S^{j}(x) \frac{\mathrm{d}^{2} w^{*}}{\mathrm{~d} x^{2}}\right)+\lambda \ell^{j+2} \frac{\mathrm{d}^{2} w^{*}}{\mathrm{~d} x^{2}}+c \ell^{j+4} w^{*}=0, \quad 0<x<1 \\
& w^{*}(1)=e^{-i \alpha} w^{*}(0), \quad\left(\frac{\mathrm{d} w^{*}(1)}{\mathrm{d} x}\right)=e^{-i \alpha}\left(\frac{\mathrm{d} w^{*}(0)}{\mathrm{d} x}\right) \\
& m^{*}(1)=e^{-i \alpha} m^{*}(0), \quad\left(\frac{\mathrm{d} m^{*}(1)}{\mathrm{d} x}\right)=e^{-i \alpha}\left(\frac{\mathrm{d} m^{*}(0)}{\mathrm{d} x}\right) \quad 0 \leq \alpha \leq 2 \pi
\end{aligned}
$$

\subsection{Variational formulation}

The presented relations (89) and (90) are necessary conditions $\delta \lambda$ for extremum of the functional

$(\delta \lambda=0): \lambda=\frac{1}{\ell^{k+2}} \frac{\int_{0}^{1} S^{k}(x) \frac{\mathrm{d}^{2} w(x)}{\mathrm{d} x^{2}} \frac{\mathrm{d}^{2} w^{*}(x)}{\mathrm{d} x^{2}} \mathrm{~d} x}{\int_{0}^{1} \frac{\mathrm{d} w(x)}{\mathrm{d} x} \frac{\mathrm{d} w^{*}(x)}{\mathrm{d} x} \mathrm{~d} x}+$ 
$c \ell^{2} \frac{\int_{0}^{1} w(x) w^{*}(x) \mathrm{d} x}{\int_{0}^{1} \frac{\mathrm{d} w(x)}{\mathrm{d} x} \frac{\mathrm{d} w^{*}(x)}{\mathrm{d} x} \mathrm{~d} x} \rightarrow w(x) \in B, w^{*}(x) \in B^{*}$

where $B$ and $B^{*}$ are classes of functions satisfying boundary conditions. Consider the analysis of the functional $\lambda$ of the problem parameters $\ell, c$ and $\alpha$. To this purpose we present the functional $\lambda$ as

$\lambda=\frac{1}{\ell^{k+2}} \frac{J_{1}}{J_{3}}+c \ell^{2} \frac{J_{2}}{J_{3}}$

where

$J_{1}=\int_{0}^{1} S^{k} \frac{\mathrm{d}^{2} w}{\mathrm{~d} x^{2}} \frac{\mathrm{d}^{2} w^{*}}{\mathrm{~d} x^{2}} \mathrm{~d} x, \quad J_{2}=\int_{0}^{1} w w^{*} \mathrm{~d} x, \quad J_{3}=\int_{0}^{1} \frac{\mathrm{d} w}{\mathrm{~d} x} \frac{\mathrm{d} w}{\mathrm{~d} x}$

Suppose that we give some increments: $\ell \rightarrow \ell+\delta \ell, c \rightarrow c+\delta c, \alpha+\delta \alpha$. Then for the first variations we will have

$\delta \lambda=\delta_{\ell} \lambda+\delta_{c} \lambda+\delta_{\alpha} \lambda=\frac{\mathrm{d} \lambda}{\mathrm{d} \ell} \delta \ell+\frac{\mathrm{d} \lambda}{\mathrm{d} c} \delta c+\frac{\mathrm{d} \lambda}{\mathrm{d} \alpha} \mathrm{d} \alpha$

Performing the variationals of the functional we obtain

$\frac{\mathrm{d} \lambda \ell}{\mathrm{d} \ell}=-\frac{k+2}{\ell^{k+3}} \frac{J_{1}}{J_{3}}+2 c \ell \frac{J_{2}}{J_{3}}$

$\frac{\mathrm{d} \lambda_{c}}{\mathrm{~d} c}=\ell^{2} \frac{J_{2}}{J_{3}}$

Using the transformation

$e^{-i \alpha}=e^{2 \pi i} e^{-i \alpha}=e^{i(2 \pi-\alpha)}$ 
we find that the solution $w^{*}(x, \alpha)$ corresponds to the solution $w(x, 2 \pi-\alpha)$ and thus we will have

$w^{*}(x ; \alpha)=w(x ; 2 \pi-\alpha)$.

Using this relation we transform the presentation of the functional (91) to the following form

$\lambda(\alpha)=\frac{1}{\ell^{k+2}} \frac{\int_{0}^{1} S^{k} \frac{\mathrm{d}^{2} w}{\mathrm{~d} x^{2}}(x ; \alpha) \frac{\mathrm{d}^{2} w}{\mathrm{~d} x^{2}}(x ; 2 \pi-\alpha) \mathrm{d} x}{\int_{0}^{1} \frac{\mathrm{d} w(x ; \alpha)}{\mathrm{d} x} \frac{\mathrm{d} w(x ; 2 \pi-\alpha)}{\mathrm{d} x} \mathrm{~d} x}+$

$c \ell^{2} \frac{\int_{0}^{1} w(x ; \alpha) w(x ; 2 \pi-\alpha) \mathrm{d} x}{\int_{0}^{1} \frac{\mathrm{d} w(x ; \alpha)}{\mathrm{d} x} \frac{\mathrm{d} w(x ; 2 \pi-\alpha)}{\mathrm{d} x} \mathrm{~d} x}$

where it is followed that $\lambda(\alpha)=\lambda(2 \pi-\alpha)$. As a result we conclude that eigenvalue $\lambda(\alpha)$ correspond the eigenfunctions $w(x, \alpha)$ and $w^{*}(x, \alpha)=w(x, 2 \pi-\alpha)$ and consequently $\lambda(\alpha)$ is double valued and also

$\lambda(\alpha)=\lambda(2 \pi-\alpha), \quad w(x, \alpha)=w^{*}(x, 2 \pi-\alpha), \quad 0 \leq \alpha \leq 2 \pi$.

In general case $w(x, \alpha)=c w^{*}(1-x, \alpha)$, where $c \neq 0$ arbitrary constant, but in our considered problem we can take $c=1$ and thus

$w(x, \alpha)=w^{*}(1-x, \alpha), \quad 0 \leq x \leq 1, \quad 0 \leq \alpha<2 \pi$

Using the (100) we construct the solution of spectral problem at $0 \leq x \leq 0.5$. To formulate the solution

$w(x, \alpha)=u(x, \alpha)+i v(x, \alpha)$ 
where $u(x, \alpha)$ and $v(x, \alpha)$ are real and imaginary parts of the $w(x, \alpha)$ we represent $w(x, \alpha)$ in the form

$u(x, \alpha)=u(1-x, \alpha), \quad 0 \leq x \leq 1, \quad 0 \leq \alpha \leq 2 \pi$

$v(x, \alpha)=-v(1-x, \alpha), \quad 0 \leq \alpha<2 \pi, \quad 0 \leq \alpha \leq 2 \pi$

Thus $u(x, \alpha)$ is even function of $x$ and $v(x, \alpha)$ is odd function.

Using these conditions and corresponding boundary conditions and the property $S(x)=S(1-x)$ we represent the variation of functional as

$\delta_{\lambda}=\frac{1}{J_{3}}\left[\frac{1}{\ell^{k+2}}\left(\left.m \delta \frac{\mathrm{d} w^{*}}{\mathrm{~d} x}\right|_{0} ^{1}+\left.m^{*} \delta \frac{\mathrm{d} w}{\mathrm{~d} x}\right|_{0} ^{1}-\left.\frac{\mathrm{d} m^{*}}{\mathrm{~d} x} \delta w\right|_{0} ^{1}-\right.\right.$

$$
\left.\left.-\left.\frac{\mathrm{d} m}{\mathrm{~d} x} \delta w^{*}\right|_{0} ^{1}\right)-\lambda\left(\left.\frac{\mathrm{d} w^{*}}{\mathrm{~d} x} \delta w\right|_{0} ^{1}+\left.\frac{\mathrm{d} w}{\mathrm{~d} x} \delta w^{*}\right|_{0} ^{1}\right)\right]
$$

Let $w(x, \alpha+\delta x)$ and $w^{*}(x, \alpha+\delta x)$ are solutions of the spectral problems (89) and (90) corresponding to the parameter $\alpha+\delta \alpha$ and

$\delta w(x, \alpha)=\frac{\mathrm{d} w(x, \alpha)}{\mathrm{d} x} \delta \alpha, \quad \delta w^{*}(x, \alpha)=\frac{\mathrm{d} w^{*}(x, \alpha)}{\mathrm{d} x} \delta \alpha$ 
are variations of these functions corresponding to parameter variation $\delta \alpha$. For variations of boundary conditions in (89) and (90) we have

$\delta_{\alpha} w(1)=i e^{i \alpha} w(0) \delta \alpha, \quad \delta_{\alpha} w^{*}(1)=i e^{-i \alpha} w^{*}(0) \delta \alpha$,

$\delta_{\alpha}\left(\frac{\mathrm{d} w(1)}{\mathrm{d} x}\right)=i e^{i \alpha}\left(\frac{\mathrm{d} w(0)}{\mathrm{d} x}\right) \delta \alpha, \quad \delta_{\alpha}\left(\frac{\mathrm{d} w(1)}{\mathrm{d} x}\right)=i e^{-i \alpha}\left(\frac{\mathrm{d} w(0)}{\mathrm{d} x}\right) \delta \alpha$.

Using presented expressions (106) for boundary variations and general representation (104) for variation of functional $\lambda$ we obtain the following formula of functional sensitivity with respect to parameter $\alpha$

$\delta_{\alpha} \lambda=\frac{\mathrm{d} \lambda}{\mathrm{d} \alpha} \delta \alpha$

$\frac{\mathrm{d} \lambda}{\mathrm{d} \alpha}=-\frac{2}{J_{3}} \operatorname{Im}\left[\frac{1}{\ell^{j+2}}\left(m^{*}(0, \alpha) \frac{\mathrm{d} w}{\mathrm{~d} x}(0, \alpha)-\frac{\mathrm{d} m}{\mathrm{~d} x}(0, \alpha) w(0, \alpha)\right)-\right.$

$\left.-\lambda\left(\frac{\mathrm{d} w^{*}}{\mathrm{~d} x}(0, \alpha) w(0, \alpha)\right)\right]$

where $\operatorname{Im}$ is the imaginary part of the expression in brackets. In the cases $\alpha=0, \pi, 2 \pi$ the solutions of the spectral problems are real and consequently $\mathrm{d} \lambda / \mathrm{d} \alpha=0$. 


\subsection{Optimal design of continuous beams}

As it can be shown (see, for example Appendix A) the solution of stability problem for the infinite homogeneous beam resting on elastic foundation represents rigorously periodic structure. In this context it is reasonable to consider periodic distributions of rigidity characteristics in the process of beam optimization against buckling.

We will construct the optimal solution in the class of periodic bending rigidity distributions

$E I(x)=A_{j} S^{j}(x), \quad j=1,2,3 ; \quad S(x)=S(x+n \ell), \quad n=0, \pm 1, \pm 2$.

with unknown before handed period $\ell$. We assume also that the optimal solution is symmetric

$S(x)=S(\ell-x)$

As it was shown previously the original buckling problem for infinite beam can be formulated at finite (unit) interval and described by (89) using the variables (88). Corresponding variational formulation at unit interval is given by (91)-(93).

The problem of optimization against buckling consists in determining of periodic distribution of beam "thickness", the value of period $\ell$ and parameter $\alpha$, such that

$\lambda(S, \ell, \alpha) \rightarrow \max _{S(x), \ell, \alpha}$ 
under volume constraint

$\int_{0}^{1} S(x) \mathrm{d} x=1$.

Performing variations of the optimized functional $\lambda$ with respect to $S(x)$ we will have the following necessary optimality conditions

$m(x) m^{*}(x)=S^{j+1}(x), \quad j=1,2,3$

$m(x)=S^{j}(x) \frac{\mathrm{d}^{2} w(x)}{\mathrm{d} x^{2}}$

Note that for symmetric distribution $S(x)=S(1-x)$ the following equalities are correct

$w(x) \equiv w^{*}(1-x), \quad m(x)=m^{*}(1-x)$.

If the thickness distribution $S(x)$ and the parameter $\alpha$ are fixed then the variation of the optimizing functional with respect to $\ell$ is reduced to calculation of derivative of the functional with respect to this parameter, while extremal values of $\ell$ are found with the help of equation $\mathrm{d} \lambda(\ell) / \mathrm{d} \ell=0$ and represented as

$\ell^{*}=\left(\frac{j+2}{2 c}\right)^{1 / j+4}\left(\frac{J_{1}}{J_{2}}\right)^{1 / j+4}$

$\lambda^{*}=\frac{j+4}{2 J_{3}}\left(\frac{2 c}{j+2}\right)^{\frac{j+2}{j+4}}\left(J_{1}^{2} J_{2}^{j+2}\right)^{1 / j+4}$. 
Wave generated periodic structure is described by spectral problem (89) and represented in the form

$w(x)=w^{0}(x) e^{i \alpha x}, \quad w^{0}(x) \equiv w^{0}(x+n \ell), \quad n=0, \pm 1, \pm 2, \ldots$

From this representation it follows that $w(n)=e^{i \alpha} w(n-1)$ and $w(1)=e^{i \alpha} w(0)$ in particular case when $n=1$. In the considered problem of optimal design the period of thickness distribution coincides with the beam halfwave length. Thus $-\alpha \ell=\pi$ or $-\alpha=\pi$ (in dimensionless form).

Formulation of the spectral problem (89) for $\alpha=\pi$ is written as

$$
\frac{\mathrm{d}^{2}}{\mathrm{~d} x^{2}}\left(S^{j}(x) \frac{\mathrm{d}^{2} w}{\mathrm{~d} x^{2}}\right)+\lambda \ell^{j+2} \frac{\mathrm{d}^{2} w}{\mathrm{~d} x^{2}}+c \ell^{j+4} w=0, \quad 0<x<1
$$

$w(1)=-w(0), \quad\left(\frac{\mathrm{d} w(1)}{\mathrm{d} x}\right)=-\left(\frac{\mathrm{d} w(0)}{\mathrm{d} x}\right)$

$m(1)=-m(0), \quad\left(\frac{\mathrm{d} m(1)}{\mathrm{d} x}\right)=-\left(\frac{\mathrm{d} m(0)}{\mathrm{d} x}\right)$

Using the property $w(x) \equiv w^{*}(1-x)$ and the representation $w(x)=u(x)-i v(x)$ we conclude that $u(x)=u(1-x)$ (symmetric function) and $v(x)=-v(1-x)$ (antisymmetric function). In what follows we present deflections in the form $w(x)=w^{s}(x)+i w^{a}(x)$, where $w^{s}(x)$ and $w^{a}(x)$ are respectively symmetric and antisymmetric functions. 
For symmetric solution the boundary conditions in the equation (113) are transformed to the form

$w^{s}(0)=0, \quad w^{s}(1)=0, \quad m^{s}(0)=0, \quad m^{s}(1)=0$

and correspond to simple supported conditions.

Thus the symmetric solutions of spectral problem (113) correspond to the symmetric buckling shapes of simply supported beam of the length $\ell$. Note that full analysis and determination of optimal solution were given in section 5 .

Consider now the antisymmetric solution and show that the solutions of optimization problem coincide for both cases. For antisymmetric solutions the boundary conditions in the equation 113 are reduced to

$\left(\frac{\mathrm{d} w^{a}(0)}{\mathrm{d} x^{2}}\right)=0, \quad\left(\frac{\mathrm{d} w^{a}(1)}{\mathrm{d} x^{2}}\right)=0, \quad\left(\frac{\mathrm{d} m^{a}(0)}{\mathrm{d} x^{2}}\right)=0, \quad\left(\frac{\mathrm{d} m^{a}(1)}{\mathrm{d} x^{2}}\right)=0$,

while the conditions

$w^{a}(1)=-w^{a}(0), \quad\left(\frac{\mathrm{d}^{2} w^{a}(1)}{\mathrm{d} x^{2}}\right)=-\left(\frac{\mathrm{d}^{2} w(0)}{\mathrm{d} x^{2}}\right)$

are automatically satisfied as it follows from definition of antisymmetric solution $w^{a}(x)=-w^{a}(1-$ $x)$. 


\section{NOTES AND CONCLUSIONS}

It is possible to analyse also the class of antisymmetric solutions and to show that the optimization problem solutions are the same for both classes (symmetric and antisymmetric functions).

Presented analysis and construction of optimal solutions for simply supported beams of the finite length are full applicable for analysis and optimization against buckling of infinite beam.

As a result of investigations we conclude that the optimal thickness distribution maximizing the critical buckling load of infinite beam resting on elastic foundation is a periodic structure with the period equal to the critical length of the finite compressed beam lying on elastic foundation with simply supported ends and optimal thickness distribution. These findings can be utilized when analyzing and optimizing a number of practical situations in production facilities.

\section{FUNDING}

The work was supported by the Academy of Finland [GrantNo. 310391,290730]

\section{REFERENCES}

Avetisyan, A., Belubekyan, M., Ghazaryan, K. (2015). Stability of a beam with periodic supports. Mechanics. Proceedings of National Academy of Sciences of Armenia 68(3):16-21.

Banichuk, N., Barsuk, A., Ivanova, S., Jeronen, J., Makeev, E., Tuovinen, T. (2016). Vibrations of a continuous web on elastic supports. Mechanics Based Design of Structures and Machines, (just-accepted). 
De Angelis, F. (2012). On the stability of discrete models of compressed beams in elastic media. In Applied Mechanics and Materials, Trans Tech Publ. Vol. 152, pp. 982-989.

De Angelis, F., Cancellara, D. (2012). On the influence of the elastic medium stiffness in the buckling behavior of compressed beams on elastic foundation. In Applied Mechanics and Materials, Trans Tech Publ, vol. 166, pp. 776-783.

Euler, L. (1766). De motu vibratorio tympanorum. Novi Commentarii academiae scientiarum imperialis Petropolitanae, 10:243-260.

Griffiths, D., Bee, G. (2014). Analytical and numerical observations on the hetényi solution for buckling of beams on elastic foundations. Journal of Engineering Mechanics, 141(1):06014014.

Moses, E., Ryvkin, M., Fuchs, M. (2001). A fe methodology for the static analysis of infinite periodic structures under general loading. Computational mechanics, 27(5):369-377.

Soldatos, K., Selvadurai, A. (1985). Flexure of beams resting on hyperbolic elastic foundations. International journal of solids and structures, 21(4):373-388.

Timoshenko, S. P. and Gere, J. M. (1961). Theory of Elastic Stability. McGraw-Hill. ISBN 9780486472072. Reprint by Dover Publications, Inc., 2009.

Timoshenko, S. P. and Woinowsky-Krieger, S. (1959). Theory of plates and shells. New York : Tokyo : McGraw-Hill, 2nd edition. ISBN 0-07-085820-9.

Vaz, M. A., Cyrino, J. C. R., Lourenço, V. E. (2014). Thermal buckling of beams on elastic foundation. In Encyclopedia of Thermal Stresses, Springer, pp. 4884-4897.

von Kármán, T. and Biot, M. A. (1940). Mathematical Methods in Engineering. McGraw-Hill. 
Yayli, M. Ö. (2017). Buckling analysis of euler columns embedded in an elastic medium with general elastic boundary conditions. Mechanics Based Design of Structures and Machines, (just-accepted).

\section{APPENDIX A}

\section{Stability of infinite beams with constant rigidity characteristics}

In what follows we present an explicit solution of the buckling problem for infinite (continuous) beam resting on a linearly elastic foundation. Instead of boundary conditions, used for beams of finite length, we apply the constraint

$|w(x)|<C \neq \infty, \quad-\infty<x<\infty$

In this case the ordinary differential equation

$E I \frac{\mathrm{d}^{4} w(x)}{\mathrm{d} x^{4}}+P \frac{\mathrm{d}^{2} w(x)}{\mathrm{d} x^{2}}+c w(x)=0, \quad-\infty<x<\infty$

describing the bending of the beam, and its fundamental solution

$w(x)=e^{i \beta x}$

are reduced to characteristic equation

$\beta^{4}-\frac{P}{E I} \beta^{2}-\frac{c}{E I}=0$ 
with respect to parameter $\beta$. Corresponding solutions of this equation can be written as

$$
\begin{aligned}
& \beta_{1,2}= \pm\left(\frac{P}{2 E I}+\left(\left(\frac{P}{2 E I}\right)^{2}-\frac{c}{E I}\right)^{1 / 2}\right)^{1 / 2} \\
& \beta_{3,4}= \pm\left(\frac{P}{2 E I}-\left(\left(\frac{P}{2 E I}\right)^{2}-\frac{c}{E I}\right)^{1 / 2}\right)^{1 / 2}
\end{aligned}
$$

As follows from the equation (115) the value of $\beta_{i} i=(1,2,3,4)$ are real and consequently we obtain the following estimate

$P \geq 2 \sqrt{c E I}$

for critical buckling forces. From equation (118) and condition for critical force $P_{*}$

$$
\begin{aligned}
& P(\beta)=E I \beta^{2}+\frac{c}{\beta^{2}} \\
& P_{*}: \quad P(\beta) \rightarrow \min _{\beta}
\end{aligned}
$$

we will have

$$
\beta_{*}=\left(\frac{c}{E I}\right)^{1 / 4}, \quad P_{*}=2(c E I)^{1 / 2}
$$

If $P=P_{*}$ then the expressions (119) take values

$$
\beta_{1}=\beta_{3}=\left(\frac{P_{*}}{2 E I}\right)^{1 / 2}=\beta_{*}
$$


$\beta_{2}=\beta_{4}=-\left(\frac{P_{*}}{2 E I}\right)^{1 / 2}=-\beta_{*}$

Thus the roots of characteristic equation (118) are double and real. Corresponding general solution is written as

$w(x)=\left(C_{1}+C_{2} x\right) e^{i \beta x}+\left(C_{3}+C_{4} x\right) e^{-i \beta_{*} x}$

Using constraint (115) we obtain $C_{2}=C_{4}=0$ and reduce (126) to the expression

$w(x)=C_{1} e^{i \beta_{*} x}+C e_{3}^{-i \beta x}=A \sin \left(\beta_{*} x+\varphi\right)$

with two arbitrary constants $A$ (amplitude) and $\varphi$ (phase). Taking into account that the considered beam is infinite and consequently we can apply arbitrary shift of coordinate origin we assume $\varphi=0$. Thus, we have

$w(x)=A \sin \left(\beta_{*} x\right)$

If $x=\ell_{*}$ and $\beta_{*} \ell_{*}=\pi$ we find from (124) the following representation for the critical parameter $\ell_{*}:$

$\ell_{*}=\frac{\pi}{\beta_{*}}=\pi\left(\frac{E I}{c}\right)^{1 / 2}$

corresponding to the critical beam length, introduced before in the case of buckling with one semiwave. 
Note that in the points

$x=x_{k}=k_{*} \ell_{*}=k \pi \quad(k=0, \pm 1, \pm 2, \ldots)$

deflections and bending moments are equal zero, i.e.

$w\left(x_{k}\right)=0, \quad m\left(x_{k}\right)=E I\left(\frac{\mathrm{d}^{2} w}{\mathrm{~d} x^{2}}\right)_{x=x_{k}}=0$

and consequently we can consider an infinite beam as a system of beams of the length $\ell$ simply supported at the nodes $x=x_{k}$. 
Table 1: Values for the functional $\Phi_{j}(w)$, critical beam lengths and critical buckling forces when $n=1,2,3, \ldots$ and $j=0,1,2,3$.

\begin{tabular}{cccc}
\hline$j$ & $\ell_{n}^{*}$ & $\Phi_{j}\left(w_{n}\right)$ & $\lambda_{n}^{*}$ \\
0 & $\frac{n \pi}{c^{1 / 4}}$ & 1 & $2 \sqrt{c}$ \\
1 & $\left(\frac{3}{2 c} n^{4} \pi^{4}\right)^{1 / 5}$ & $\frac{1}{\left(n^{2} \pi^{2}\right)^{1 / 5}}$ & $\frac{5}{2}\left(\frac{8 c^{3}}{27 n^{2} \pi^{2}}\right)^{1 / 5}$ \\
2 & $\left(\frac{2}{c}\right)^{1 / 6}\left(n^{2} \pi^{2}\right)^{1 / 3}$ & $\frac{1}{\left(n^{2} \pi^{2}\right)^{1 / 3}}$ & $3\left(\frac{c^{2}}{4}\right)^{1 / 3} \frac{1}{\left(n^{2} \pi^{2}\right)^{1 / 3}}$ \\
3 & $\left(\frac{5}{2 c} n^{4} \pi^{4}\right)^{1 / 7}$ & $\frac{1}{(n \pi)^{6 / 7}}$ & $\frac{7}{2}\left(\frac{2 c}{5}\right)^{5 / 7} \frac{1}{(n \pi)^{6 / 7}}$ \\
\hline
\end{tabular}




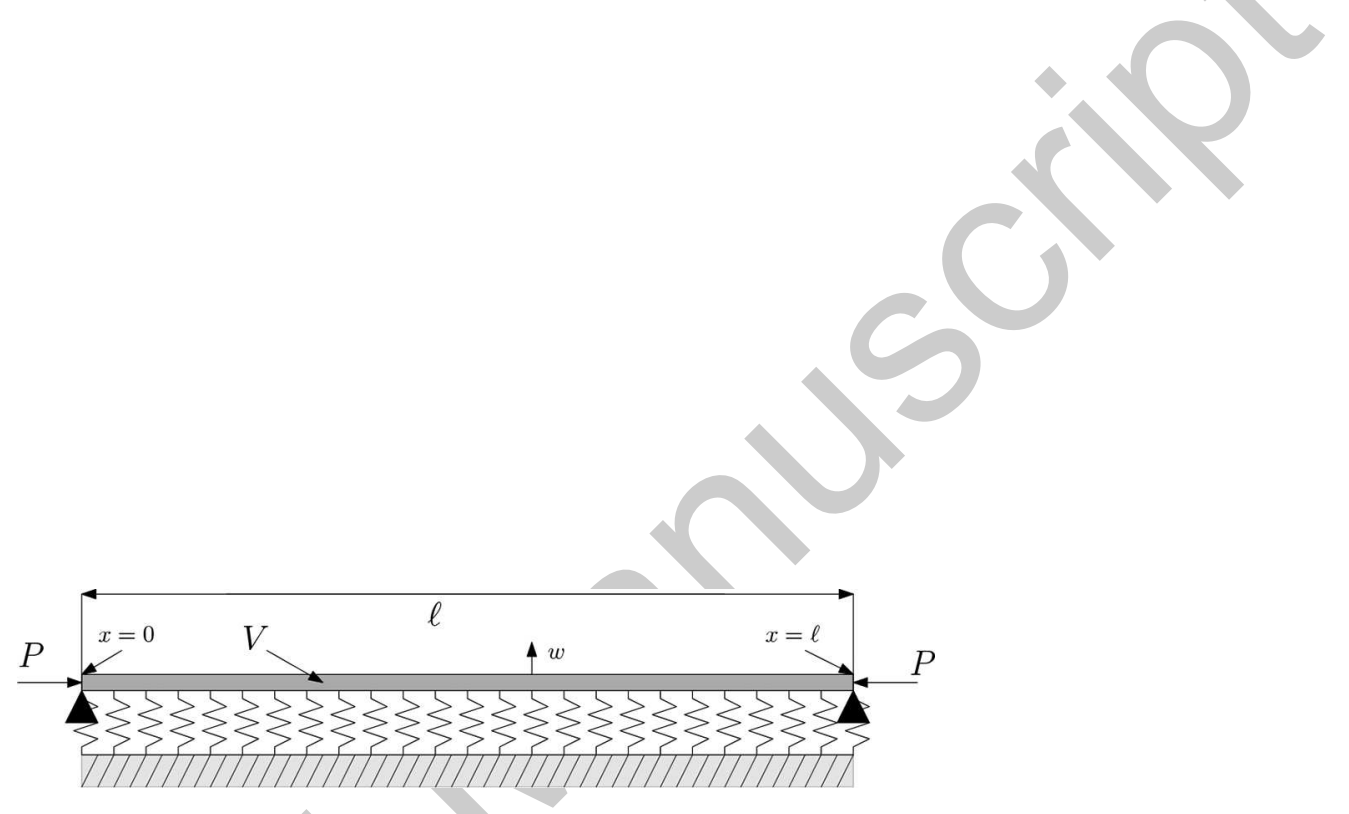

Figure 1: A finite elastic beam on linearly elastic foundation. 


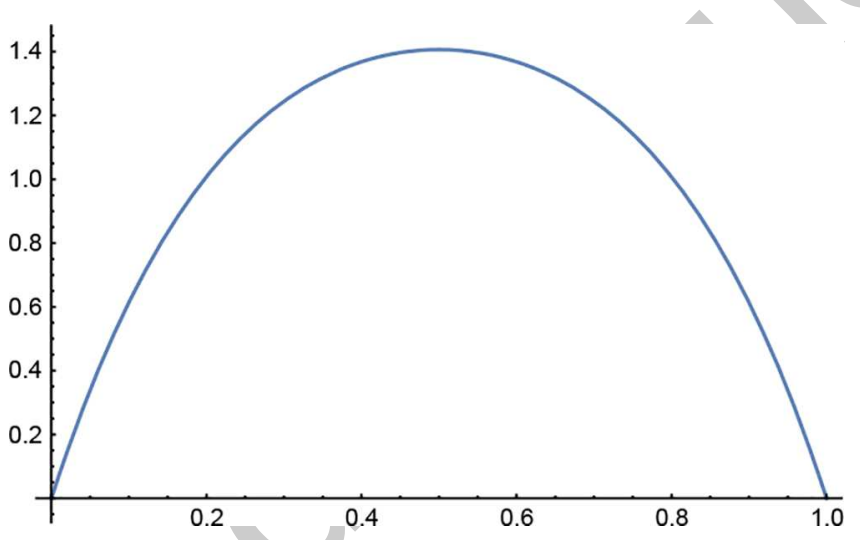

Figure 2: Optimal cross-sectional area distribution $S(x)(\alpha=1)$. 


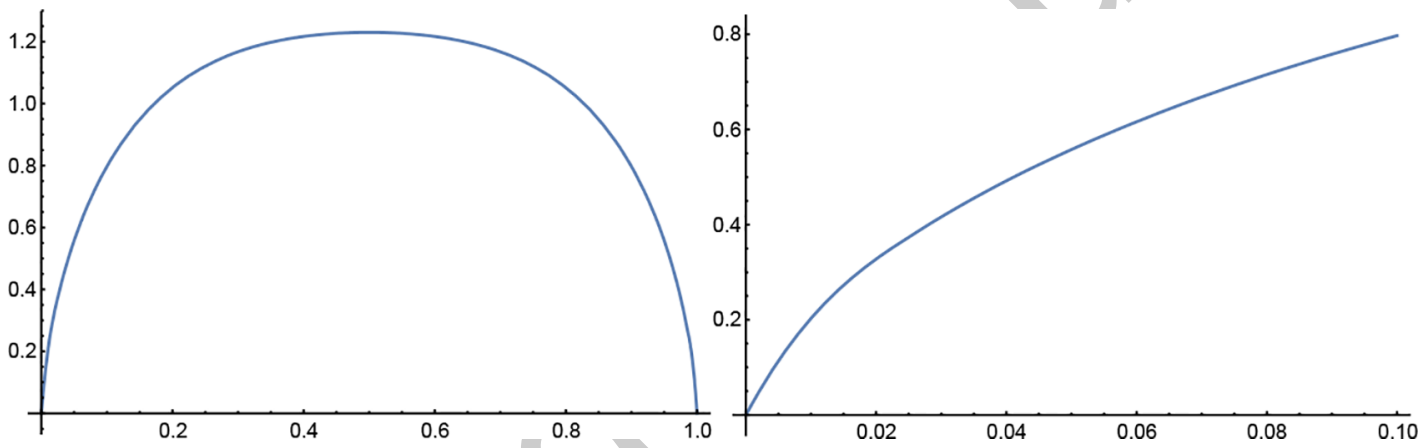

Figure 3: Optimal cross-section area distribution $S(x)$ when $\alpha=2$. 


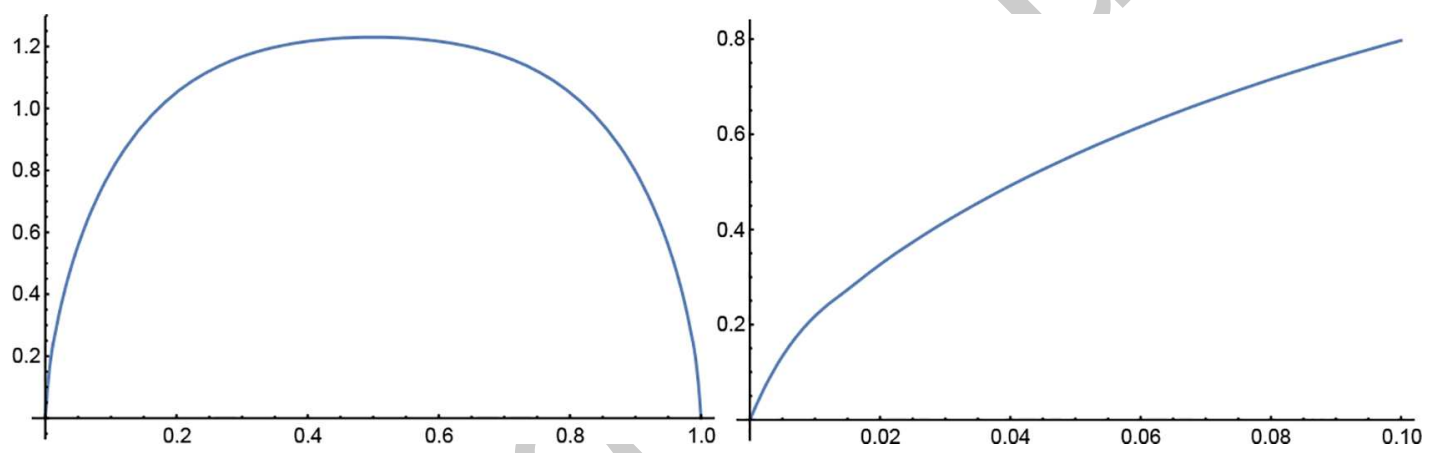

Figure 4: Optimal cross-section area distribution $S(x)$ when $\alpha=3$. 